\title{
Perivascular Cells and NADPH Oxidase Inhibition Partially Restore Hyperglycemia-Induced Alterations in Hematopoietic Stem Cell and Myeloid-Derived Suppressor Cell Populations in the Bone Marrow
}

\author{
Ji-Young Kim ${ }^{1}$, Ji Yoon Lee ${ }^{2}$, Kwon-Soo Ha ${ }^{3}$, Eun-Taek Han ${ }^{4}$, Won Sun Park ${ }^{5}$, Chang-Ki Min ${ }^{6,7}$, Seok-Ho Hong ${ }^{1}$ \\ ${ }^{1}$ Department of Internal Medicine, School of Medicine, Kangwon National University, Chuncheon, Korea \\ ${ }^{2}$ Department of Biomedical Sciences, Stem Cell Institute, CHA University, Seongnam, Korea \\ ${ }^{3}$ Department of Molecular and Cellular Biochemistry, School of Medicine, Kangwon National University, Chuncheon, Korea \\ ${ }^{4}$ Department of Medical Environmental Biology and Tropical Medicine, School of Medicine, Kangwon National University, \\ Chuncheon, Korea \\ ${ }^{5}$ Department of Physiology, School of Medicine, Kangwon National University, Chuncheon, Korea \\ ${ }^{6}$ Department of Hematology, Seoul St. Mary's Hospital, The Catholic University of Korea, Seoul, Korea \\ ${ }^{7}$ Leukemia Research Institute, The Catholic University of Korea, Seoul, Korea
}

Background and Objectives: Patients suffer from long-term diabetes can result in severe complications in multiple organs through induction of vascular dysfunctions. However, the effects of chronic hyperglycemic conditions on hematopoiesis and the microenvironment in the bone marrow (BM) are not yet well understood.

Methods: BM cells were harvested from femurs of mice and analyzed using flow cytometry. Human PVCs were cultured in serum-free $\alpha$-MEM. After 24hrs, PVC-CM was collected and filtered through a $0.22 \mu \mathrm{m}$ filter.

Results: In this study, we showed that hyperglycemia alters hematopoietic composition in the BM, which can partially be restored via paracrine mechanisms, including perivascular cells (PVCs) and NADPH oxidase (NOX) inhibition in mice with streptozotocin-induced diabetes. Prolonged hyperglycemic conditions resulted in an increase in the frequency and number of long-term hematopoietic stem cells as well as the number of total BM cells. The altered hematopoiesis in the BM was partially recovered by treatment with PVC-derived conditioned medium (CM). Long-term diabetes also increased the number of myeloid-derived suppressor cells in the BM, which was partially restored by the administration of PVC-CM and diphenyleneiodonium (DPI), a NOX inhibitor. We further showed the downregulation of ERK and p38 phosphorylation in BM cells of diabetic mice treated with PVC-CM and DPI. This may be associated with dysfunction of hematopoietic cells and promotion of subsequent diabetic complications.

Conclusions: Our data suggested that alterations in BM hematopoietic composition due to prolonged hyperglycemic conditions might be restored by improvement of the hematopoietic microenvironment and modulation of NOX activity.

Keywords: Hyperglycemia, Hematopoiesis, Perivascular niche, NOX, MDSCs

Received: October 19, 2018, Revised: October 19, 2018, Accepted: November 25, 2018, Published online: December 31, 2018

Correspondence to Seok-Ho Hong

Department of Internal Medicine, School of Medicine, Kangwon National University, 1 Kangwondaehak-gil, Chuncheon 24341, Korea

Tel: +82-33-250-7819, Fax: +82-33-244-2367, E-mail: shhong@kangwon.ac.kr

Co-Correspondence to Chang-Ki Min

Department of Internal Medicine, Seoul St. Mary's Hospital, College of Medicine, The Catholic University of Korea, 222 Banpo-daero, Seocho-gu, Seoul 06591, Korea

Tel: +82-2-2258-6053, Fax: +82-2-599-3589, E-mail: ckmin@catholic.ac.kr

(a) This is an open-access article distributed under the terms of the Creative Commons Attribution Non-Commercial License (http://creativecommons.org/licenses/by-nc/4.0/), which permits unrestricted non-commercial use, distribution, and reproduction in any medium, provided the original work is properly cited.

Copyright (c) 2019 by the Korean Society for Stem Cell Research 


\section{Introduction}

Diabetes mellitus (DM) is a group of metabolic diseases characterized by hyperglycemia and excessive urination resulting from insulin resistance or lack of insulin (1). The chronic hyperglycemic condition induces the secretion of advanced glycation end-products (AGEs) from circulating neutrophils, which increases myelopoiesis through the activation of AGE receptor on macrophages and common myeloid progenitors in the bone marrow $(\mathrm{BM})(2)$. As a result, inflammatory monocytes and macrophages increase in the BM infiltrate and migrate into target organs. This induces abnormal functions and structures in microvessels, which leads to diabetic vascular complications such as neuropathy, retinopathy, and nephropathy. Thus, circulating and BM hematopoietic stem cells (HSCs) are not only target organs, but are also considered as a contributor to chronic diabetic complications.

In recent years, multiple lines of evidence suggest that diabetes compromises the structure and function of the BM microenvironment. A reduced number of circulating endothelial progenitor cells and HSCs were observed in diabetic human samples (3-5). In addition, diabetic patients showed poor mobilization in response to granulocyte colony-stimulating factor $(5,6)$. Similar findings have been reported in streptozotocin (STZ)-induced diabetic mice, which is mediated by decreased release of a chemoattractant molecule as well as decreased activation of endothelial nitric oxide (NO) synthase in the BM (7). Kojima et al. (8) reported BM dysfunction including increased fat deposition, reduced hematopoietic tissues, and microvascular rarefaction in diabetic individuals. Ferraro et al. (5) further observed downregulation of C-X-C Motif Chemokine Ligand 12 (CXCL12) in nestin-positive perivascular cells (PVCs). The changes in the BM microenvironment in diabetes are accompanied by functional alterations of hematopoietic cells in the BM. For example, hyperglycemia impaired the colony-forming capability of the BM (9). Downregulation of CXCL12 in perivascular niche cells resulted in the retention of HSCs and their mobilization defects in the BM of diabetic mice $(5,10)$. Furthermore, in vitro exposure of BM cells to high glucose was found to activate insulin and tumor necrosis factor- $\alpha$ transcripts $(11,12)$. As mentioned above, it is an emerging hypothesis that diabetes induces hematopoietic alteration and $\mathrm{BM}$ niche dysfunction associated with the development of diabetic complications in multiple organs. However, the crosstalk between diabetes and hematopoietic cells has been less investigated.

In our present study, we investigated if hyperglycemic condition influenced hematopoietic composition in the $\mathrm{BM}$ and further examined the regenerative potential of PVCs and NADPH oxidase (NOX) inhibition. We found that the hyperglycemic condition induced an increase in the frequency and number of long-term (LT) HSCs as well as the number of total BM cells. The altered hematopoiesis in the BM was partially recovered by the treatment of PVC-derived conditioned medium (CM). Hyperglycemic also increased the number of myeloid-derived suppressor cells (MDSCs) in the BM, which was partially restored by the administration of PVC-CM and NOX inhibitor. We further showed that treatment with PVC-CM and DPI downregulated ERK gene expression and p38 phosphorylation in BM cells of diabetic mice. These findings suggested that the altered hematopoietic composition in the $\mathrm{BM}$ by prolonged hyperglycemic conditions might be restored by improving the hematopoietic microenvironment and modulating the activity of NOX.

\section{Materials and Methods}

\section{Mice}

Mice C57BL/6J mice were purchased from Dooyeol Biotech (Seoul, Korea). Mice were housed in a specific pathogen-free facility. All animal experiments were approved by the Institutional Animal Care and Use Commitment of Kangwon National University. Male C57BL/6J mice (18 20 g, 6 weeks) were intraperitoneally injected with low doses of $50 \mathrm{mg} / \mathrm{kg} \mathrm{STZ} \mathrm{(S0130,} \mathrm{Sigma,} \mathrm{USA)} \mathrm{dai-}$ ly for 5 days to induce type $1 \mathrm{DM}$ (T1DM). After 5 days, blood glucose levels higher than $250 \mathrm{mg} / \mathrm{dl}$ were accepted as indicating diabetes induction in the mice. After induction of diabetes, mice were intravenously administered PVC-CM $(40 \mu \mathrm{g} / 100 \mu \mathrm{l})$ for 6 weeks, whereas controls received vehicle.

\section{Preparation of PVC-CM}

Human PVCs were seeded in $150 \mathrm{~cm}^{2}$ culture dishes. At $90 \%$ confluence, the cells were washed twice with phosphate buffered saline (PBS) and cultured in fresh serum-free $\alpha$-MEM. After $24 \mathrm{hrs,} \mathrm{PVC-CM}$ was collected and filtered through a $0.22 \mu \mathrm{m}$ filter. Filtered medium were concentrated using a $3-\mathrm{kDa}$ cutoff ultrafiltration membrane and stored at $-80^{\circ} \mathrm{C}$ until use (3K, Amicon).

\section{BM cells harvest and flow cytometry}

BM was harvested from femurs by flushing with ice cold RPMI. The cell suspension was centrifuged at $800 \mathrm{~g}$ for 5 min, and supernatant aspirated. The cells were resuspended in RBC lysis buffer for 3 min and adding PBS 
with $1 \%$ FBS buffer and once again centrifuged. Cells were incubated with V450 mouse lineage antibody cocktail (561301, BD), in incubation with rat anti mouse Ly-6A/E (Sca-1) (558162, BD) and Rat anti mouse CD117 (c-kit) (553356, BD) antibodies, to quantify the percentage of Lin $\mathrm{Scal}^{+} \mathrm{CKit}^{+}$(LSK) Cells. Another population of cells from BM was stained with rat anti mouse CD34 (560238, BD) to identify the population of LT-HSCs and short-term (ST)-HSCs. The antibodies used for the MDSCs experiments were CD11b-PEcy7 (25-0112-82, eBioscience), Ly6G-eflur 450 (45-5931-82, eBioscience), and Ly6C-APC (17-5932-82, eBioscience). All antibodies were incubated for $30 \mathrm{~min}$ at $4^{\circ} \mathrm{C}$ and samples were analyzed with FACS Canto II (BD).

\section{Colony-forming unit (CFU) assay}

CFU assay was performed as previously described (13). Briefly, 6,000 BM cells were plated into methylcellulose H4434 (Stem Cell Technologies) and incubated for 7 10 days at $37^{\circ} \mathrm{C}$ in $5 \% \mathrm{CO}_{2}$.

\section{Real time qRT-PCR}

Total RNA from lung tissues was extracted using an RNeasy Mini Kit (Qiagen, Duesseldorf, Germany) and the cDNA was synthesized using TOPscript ${ }^{\mathrm{TM}}$ RT DryMIX (Enzynomics, Daejeon, Korea). qPCR analyses were performed using a Step One Plus real time PCR system (Applied Biosystems, Warrington, UK) with TOPreal ${ }^{\mathrm{TM}}$ qPCR 2X PreMIX (Enzynomics). To amplify GAPDH, arginase-1 (ARG1) and inducible nitric oxide synthase (iNOS), we used the following primers: GAPDH forward (F) 5'-AACTTTGGCATTGTGGAAGG-3', reverse (R) 5'ACACATTGGGGGTAGGAACA-3', ARG1 F 5'-ATGCAAGAGACCTTCAGCTAC-3', R 5'-GCTGCTTTCCCAAGAGTTGGG-3, and iNOS F 5'-GGCAGCCTGTGAGACCTTTG-3', R 5'-TGAAGCGTTTCGGGATCTG-3'.

\section{Western blotting}

Lysates from BM total cells were lysed in protein lysis buffer with protease inhibitor (\#1860932, Thermo Scientific) and quantified using the BCA protein assay (\#23228, Thermo Scientific). The $20 \mu \mathrm{g}$ of protein were separated by SDS-PAGE using 10 15\% gel and then transferred to PVDF membranes (IPVH00010, Millipore, Billerica, MA, USA). Membranes were blocked with 5\% skim milk for $1 \mathrm{~h}$ at room temperature and then incubated with primary antibodies against anti-phospho-p44/42 MAPK (4370s, Cell Signaling), anti-p44/42 MAPK (4695s, Cell Signaling), anti-phospho-p38 MAPK (4511s, Cell Signaling), anti-p38 MAPK (9212s, Cell Signaling), anti-phospho-SAPK/
JNK (4668s, Cell Signaling), anti-SAPK/JNK (9252s, Cell Signaling) and their corresponding secondary antibodies (anti-rabbit BML-SA204-0100, anti-mouse ADI- SAB-300-J, Enzo) overnight at $4^{\circ} \mathrm{C}$ and for $1 \mathrm{~h}$ at room temperature, respectively. Membranes were scanned with ChemiDoc Imaging System (Bio-Rad Laboratories, Hercules, CA, USA).

\section{Statistical analysis}

Results are presented as means \pm SD. Statistical comparisons between groups were conducted using the Student's $t$-test and one-way ANOVA. The minimal significance level was set at $\mathrm{p}<0.05$.

\section{Results}

\section{Hyperglycemic conditions increase the number of BM cells}

To investigate the effects of hyperglycemic conditions on hematopoietic composition in the BM, type I diabetic mice were induced by daily injection of STZ for 5 days and sacrificed 4 and 6 weeks after the last injection of STZ (Fig. 1A). The STZ-injected mice exhibited gradual weight loss during the time course of experiments compared to the control mice $(24.5 \pm 1.5 \mathrm{~g}$ vs. $28.37 \pm 1.6 \mathrm{~g}$ at 6 weeks after the onset of diabetes) (Fig. 1B). The blood glucose level in diabetic mice was maintained $\geq 250 \mathrm{mg} /$ $\mathrm{dL}$ up to 100 days after the onset of diabetes (Fig. 1C). We harvested BM-derived mononuclear cells at 4 and 6 weeks after induction of diabetes and first determined the impact of hyperglycemic conditions on the total number of BM cells. The total number of BM cells was significantly increased in diabetic mice compared to the control mice at 6 weeks after induction of diabetes, while there was no difference in the number of BM cells at 4 weeks (Fig. 1D). This finding suggested that prolonged hyperglycemic conditions may alter the hematopoietic composition in the BM.

\section{Percentage and number of LT-HSCs are increased in STZ-injected mice}

We assumed that an increased number of total BM cells was reflected in the hematopoietic composition in the BM. Therefore, we first investigated the impact of hyperglycemia on the frequency and number of LT- and ST-HSC populations at 4 and 6 weeks after induction of diabetes. The frequency of LT-HSCs $\left(\mathrm{Lin}^{-} \mathrm{Scal}^{+} \mathrm{cKit}^{+}\right.$ $\mathrm{CD} 34^{-}$) was not changed at 2 weeks after induction of diabetes (data not shown) but increased at 4 weeks and was retained up to 6 weeks, whereas the frequency of ST-HSCs $\left(\mathrm{Lin}^{-} \mathrm{Scal}^{+} \mathrm{cKit}^{+} \mathrm{CD} 34^{+}\right.$) was not affected by 
A

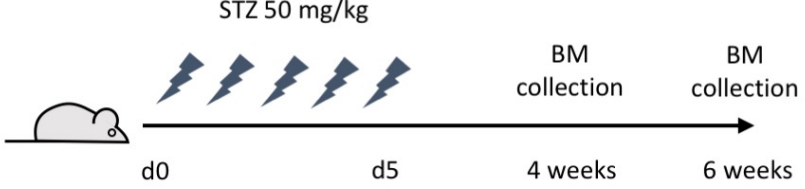

B

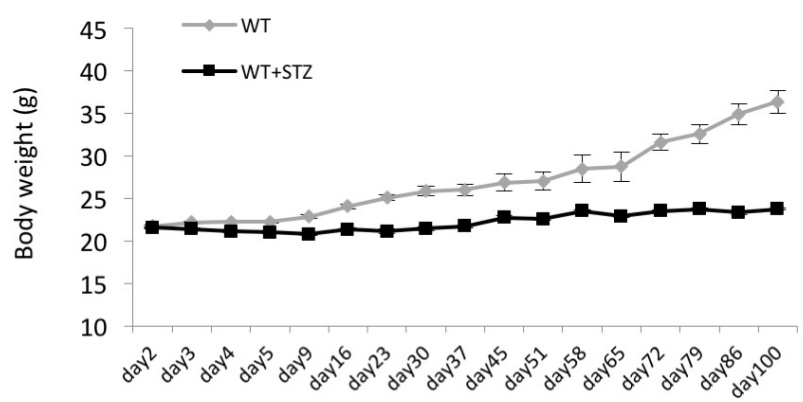

C

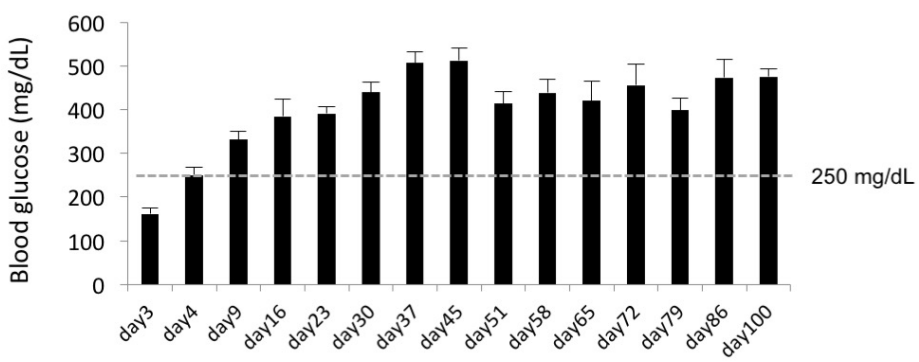

D

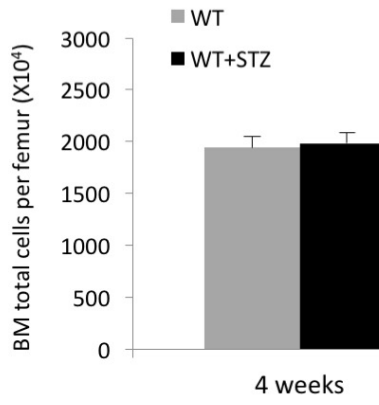

hyperglycemic conditions (Fig. 2A). We analyzed the number of LT- and ST-HSCs in the BM and similarly found an increase of the LT-HSC fraction at 4 and 6 weeks after induction of diabetes, but no changes in the ST-HSC fraction (Fig. 2B). We then determined the effects of diabetes on the colony-forming ability of BM-derived HSCs. Total numbers of colony-forming units (CFUs) and CFU subtypes were not significantly different between the STZ-injected and the control mice, while the percentage and number of CFU-E showed an increased trend without reaching significance (Fig. 2C-E). These results suggested that hyperglycemia not only affected self-renewal or proliferative activity of LT-HSCs in the $\mathrm{BM}$, but also may have influenced their functional characteristics.
Fig. 1. Changes in body-weight and blood glucose levels in STZ-treated diabetic mice and control. (A) Protocols for the induction of hyperglycemia using STZ. (B) Blood glucose and (C) body weights measurements of STZ-treated mice for 100 days. WT $(n=19)$, STZ-treated mouse $(n=26)$. (D) BM total cell counts per femurs of STZ-treated and control mice. WT $(n=5)$, STZ-treated mice $(n=8)$.
Alterations in the number of total BM cells of diabetic mice partially revert to normal by PVC-CM treatment

Previous studies indicated that diabetes impaired the structure and function of the $\mathrm{BM}$ microenvironment, which is mechanically mediated by activation of inflammatory responses and generation of reactive oxygen species (ROS) via a NOX system. Thus, we further determined if perivascular niche support and an inhibition of NOX recovered the hematopoietic alteration induced by hyperglycemic conditions. After induction of diabetes, the mice daily received either $40 \mu \mathrm{g}$ PVC-CM or $20 \mu \mathrm{g}$ diphenyleneiodonium (DPI, NOX inhibitor) for up to 6 weeks (Fig. 3A). Administration of PVC-CM or DPI did not recover the loss of weight and reduce the increased blood glucose level in STZ-treated mice (Fig. 3B). The in- 

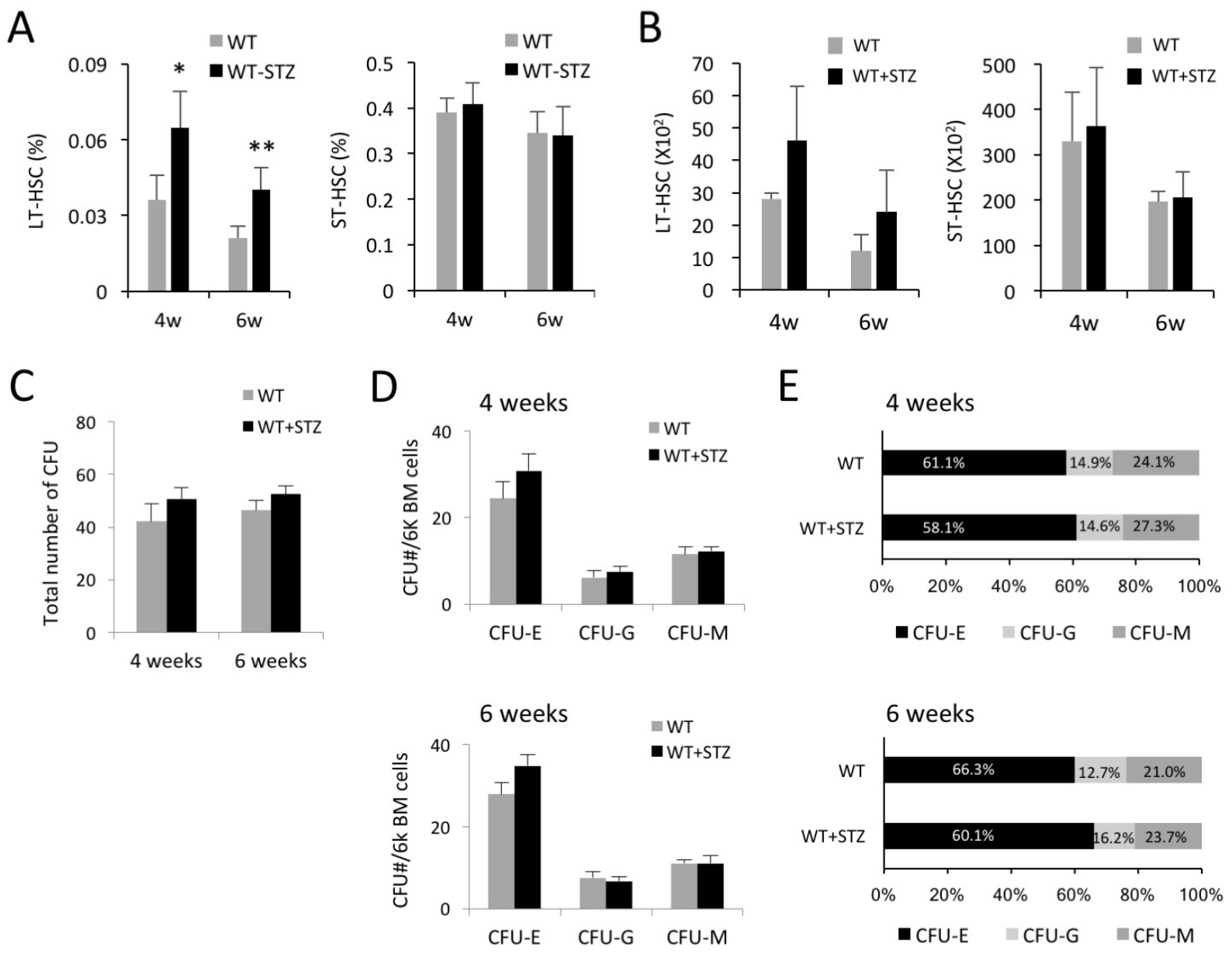

Fig. 2. Characterization of the HSCs in the BM of STZ-treated diabetic mice and control. (A) Frequency of LT-HSC and ST-HSC in different phases of STZ-treated diabetic and control mice. WT $(n=20)$, STZ-treated mouse $(n=28)$. Data are mean $\pm S D$., $* p<0.05$. (B) The cell counts of LT- and ST-HSC of BM cells in different phases of STZ-treated diabetic and control mice. (C) CFU colony numbers of BM cells in different phases of STZ-treated diabetic and control mice. (D) Number of CFU-E,-G,-M per 6000 cells of BM cells in different phases of STZ-treated diabetic and control mice. (E) Distribution of CFU-E,-G,-M as a percentage of BM cells in different phases of STZ-treated diabetic and control mice. WT ( $n=6)$, STZ-treated mice $(n=6)$.

creased number of total $\mathrm{BM}$ cells in diabetic mice was partially reverted to normal when PVC-CM was administered (Fig. 3C), whereas both PVC-CM and DPI did not reduce the increased frequency of LT-HSCs (Fig. 3D). Furthermore, an increased trend in the total number of CFUs and CFU-E in STZ-treated mice partially reverted to normal in both PVC-CM and DPI-injected mice (Fig. 3E and 3F). These results indicated that hyperglycemia may have impaired the BM microenvironment and stimulated the NOX system, which could be partially restored by perivascular support and inhibition of NOX activity.

\section{PVC-CM treatment modified the expansion of MDSCs population by hyperglycemia}

A recent study reported a key role of $\mathrm{T}$ cells in the progression of type $2 \mathrm{DM}$ (T2DM), which can be suppressed by MDSCs (14). Therefore, we next asked if the hyperglycemic condition affected the number and frequency of MDSCs in the BM. Mouse MDSCs were divided into two subtypes based on expression of surface markers as follows: $\mathrm{CD} 11 \mathrm{~b}^{+} \mathrm{Ly}_{6 \mathrm{G}}{ }^{+} \mathrm{Ly} 6 \mathrm{C}^{\text {low }}$ granulocytic MDSCs (GMDSCs) and $\mathrm{CD}_{11 \mathrm{~b}^{+} \mathrm{Ly}^{-} \mathrm{G}^{-} \text {Ly6C }}{ }^{\text {hi }}$ monocytic MDSCs (M-MDSCs) (Fig. 4A) (15, 16). We found that hyperglycemic conditions did not alter the ratio between G-MDSCs and M-MDSCs as well as the frequencies of Gand M-MDSC subsets (Fig. 4B and 4C). The treatment of PVC-CM and DPI did not influence the frequency and ratio of both cell types. Interestingly, hyperglycemic conditions significantly increased the number of G- and M-MDSCs in the BM, which was efficiently restored by the administration of PVC-CM and DPI (Fig. 4D). ARG1 and iNOS are highly produced by MDSCs $(17,18)$ and 
A
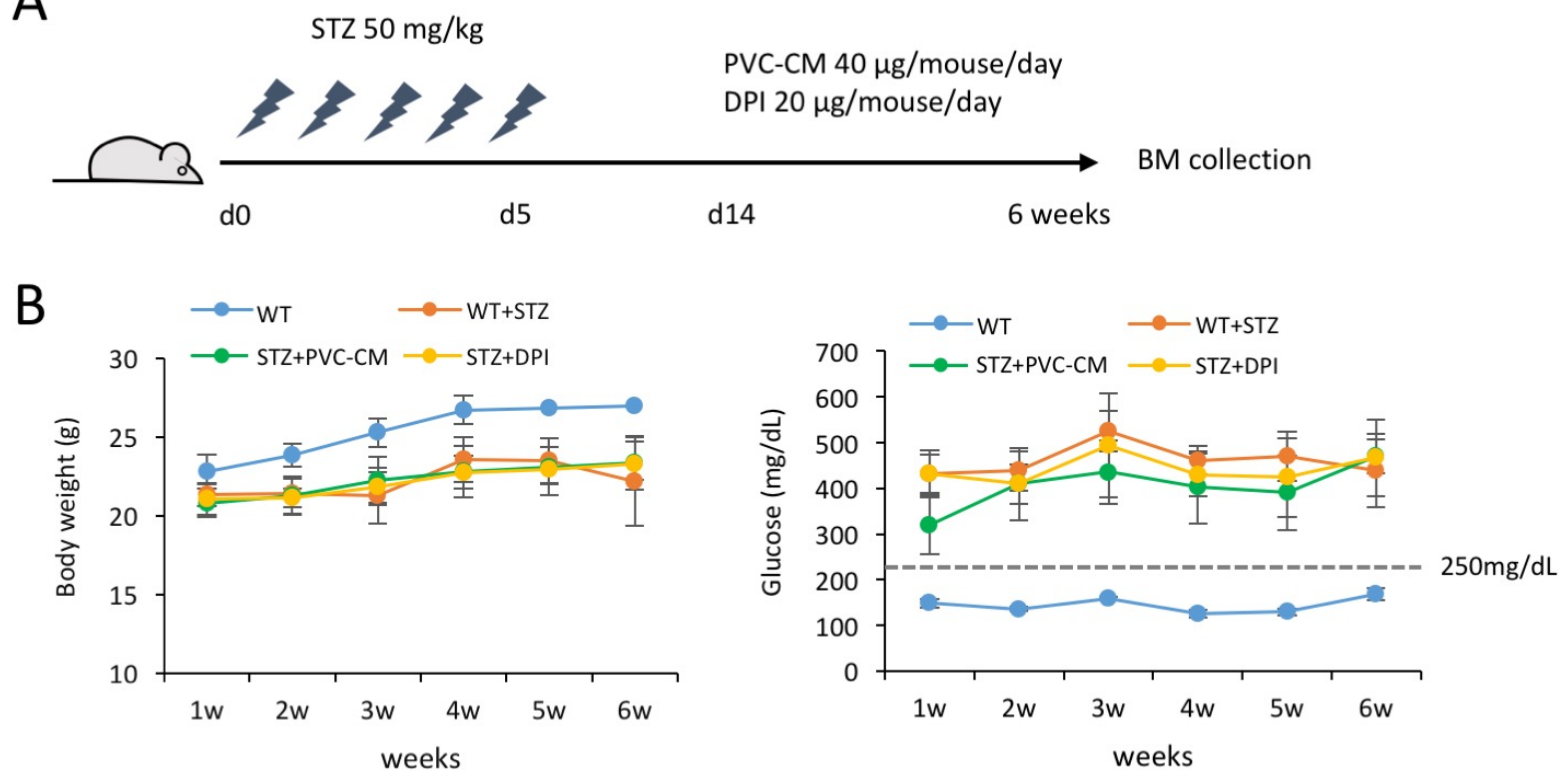

C

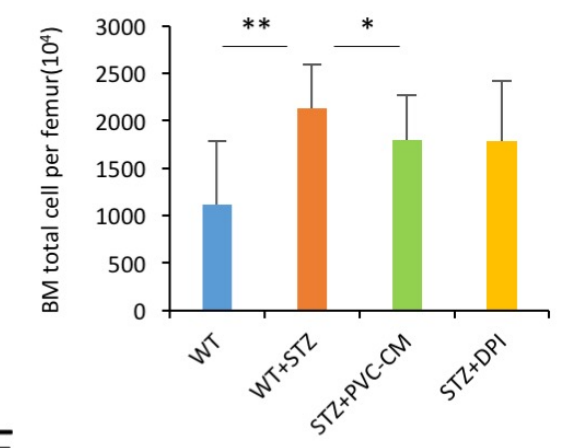

$E$

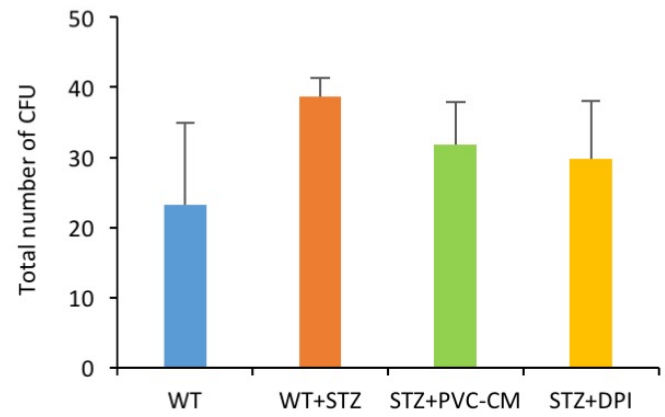

D
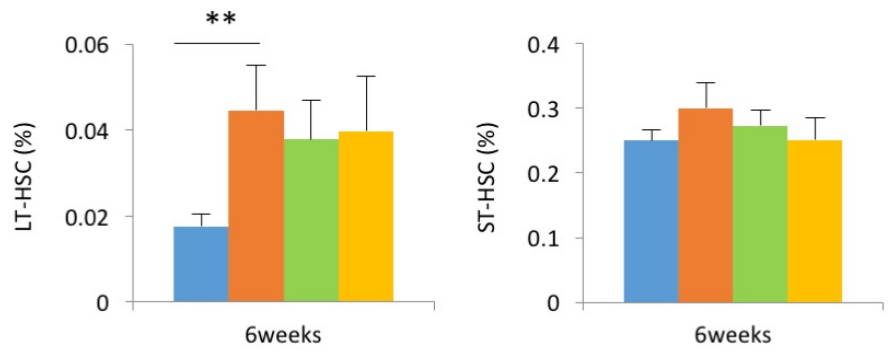

$\mathrm{F}$

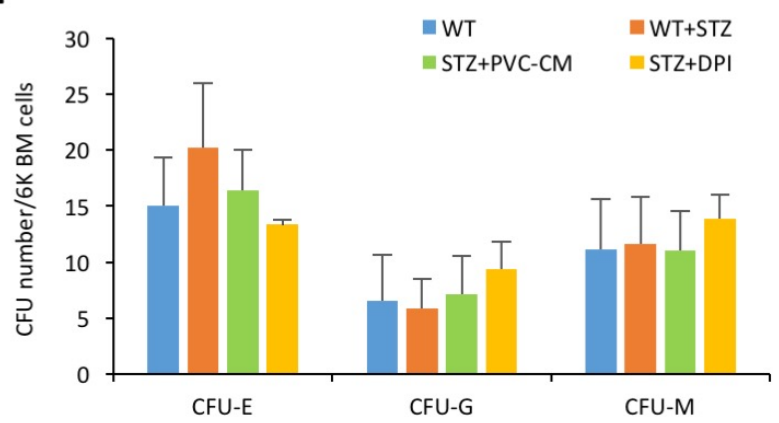

Fig. 3. Effect of PVC-CM in the hematopoietic stem cell of STZ-treated diabetic mice. (A) Protocols for the PVC-CM treatment after induction of hyperglycemia using STZ. At 6weeks after PVC-CM treatment, (B) Blood glucose and body weights measurements of STZ-treated, STZ+ PVC-CM and STZ+DPI mice. WT $(n=9)$, STZ-treated mice $(n=9)$, STZ+PVC-CM $(n=7)$, STZ+DPI $(n=8)$. (C) BM total cell counts per femurs of STZ-treated, STZ+PVC-CM, STZ+DPI and control mice. (D) Frequency of LT-HSC and ST-HSC in each BM cells. (E) CFU colony numbers of BM cells in each mouse. (F) Number of CFU-E,-G,-M per 6000 cells of BM cells in each mouse. WT ( $n=16)$, STZ-treated mice $(n=20)$, STZ + PVC-CM $(n=17), S T Z+D P I(n=17)$.

involved in immunosuppressive events in pathologic condition. Thus, we examined whether PCV-CM and DPI treatment would influence the transcript levels of ARG1 and iNOS in MDSCs. PCR data showed that their levels were decreased by PVC-CM and DPI treatment (Fig. 4E). To understand the molecular mechanism of the alterations 
A
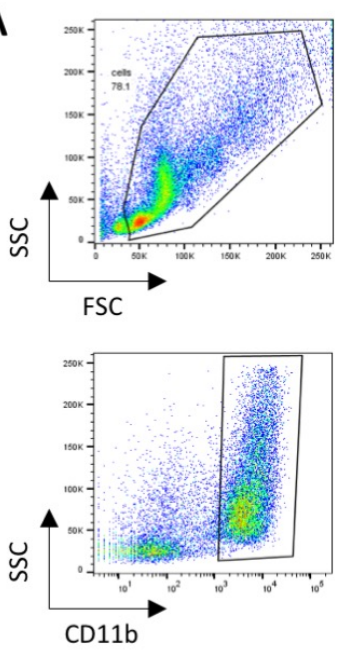

C

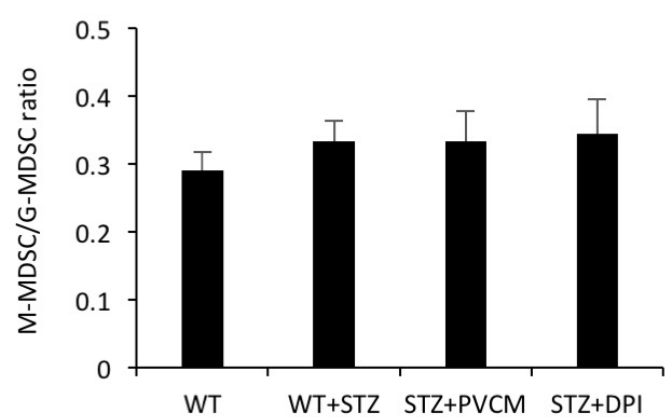

$E$
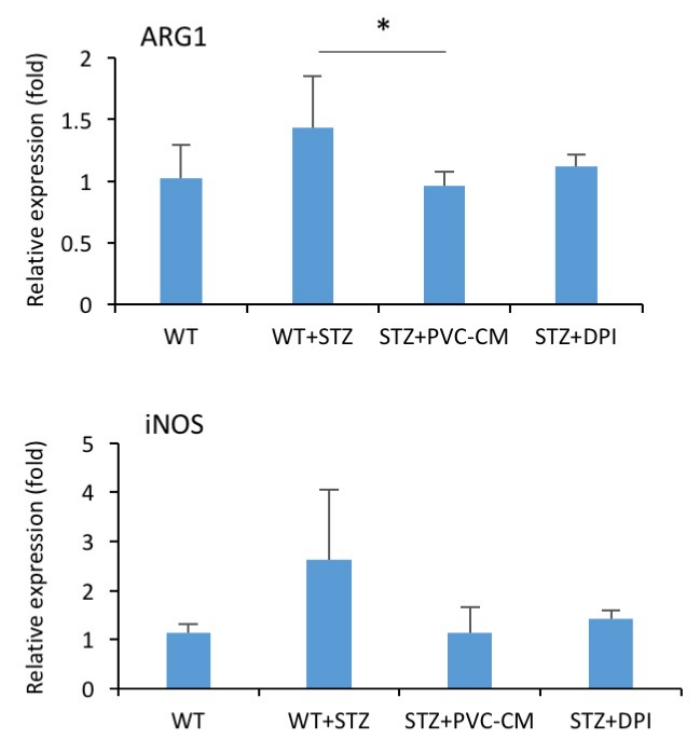

B

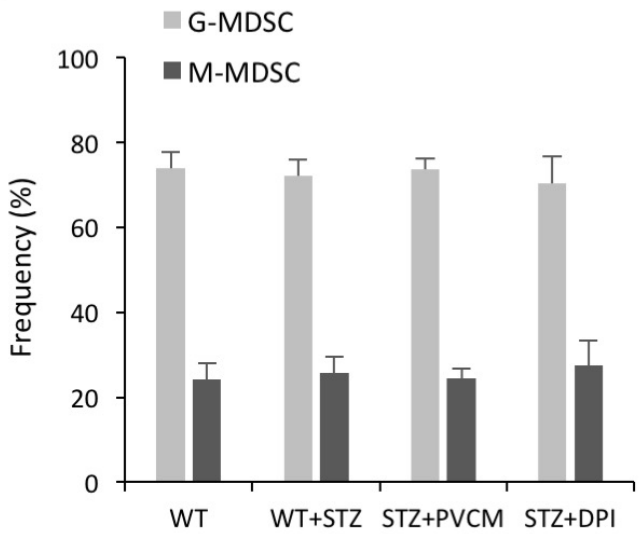

6 weeks

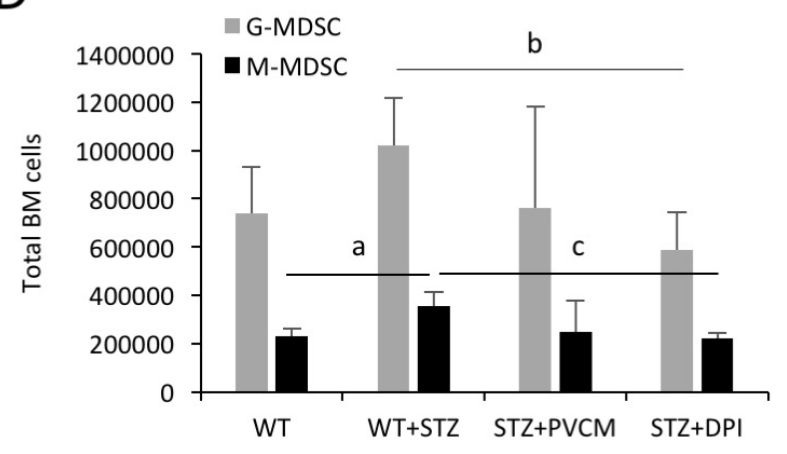

$\mathrm{F}$

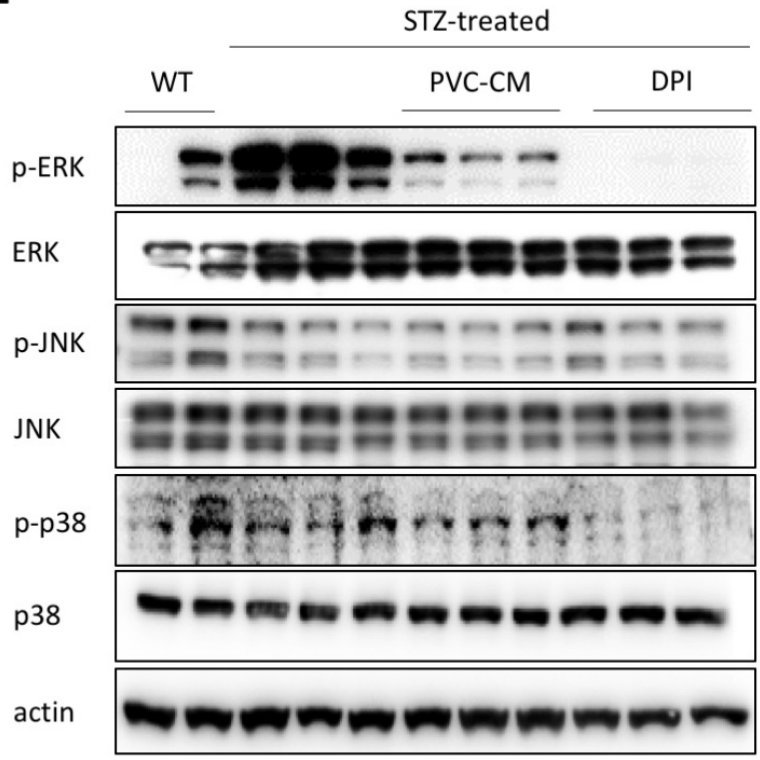

Fig. 4. PVC-CM treatment influence activated and differentiated mouse MDSCs in BM of STZ-treated diabetic mice. (A) Representative FACS plot of G-MDSCs and M-MDSCs in total BM. (B) Frequency of G-MDSC and M-MDSC in each BM cells. (C) M-MDSC/G-MDSC ratio in total BM cells of each mouse. (D) MDSC counts in total BM cells of each mouse. Data represent mean $\pm S D,{ }^{*} p<0.05,{ }^{* *} p<0.001$. (E) Expression levels of ARG1 and iNOS in total BM cells. WT $(n=5)$, STZ-treated mice $(n=7)$, STZ+PVC-CM $(n=5), S T Z+D P I(n=6)$. Data are mean \pm SD., $* \mathrm{p}<0.05$. (F) Western blot analysis for MAPK in total BM cells. 
in hematopoietic composition, we examined the expression levels of MAPKs in the BM cells of the control and diabetic mice. We found the activation of ERK and p38 phosphorylation in STZ-treated mice compared to the control mice, which was completely reversed to normal after PVC-CM and DPI treatment (Fig. 4F). However, expression of JNK was not changed by hyperglycemic conditions and PVC-CM/DPI treatment. These findings suggested that alterations in the number of MDSCs and activation of MAPK signaling molecules might be associated with functions of MDSCs and subsequent diabetic complications.

\section{Discussion}

Cellular niches in the BM are functional compartments that control the behavior of HSCs and immune cells via direct and indirect signal transmission. The results of our study showed that prolonged hyperglycemic conditions altered the hematopoietic composition in the BM, which was partially restored by the paracrine support of perivascular niche cells and modulation of NOX activity. This finding suggested that DM influenced the function and differentiation of hematopoietic cells by impairing the BM perivascular niche.

Since the concept of BM niche was first proposed (19), multiple types of niche components and their regulatory factors have been relatively well defined (20). First, the bone-forming osteoblasts secret various hematopoietic cytokines and adhesion molecules that contribute to the maintenance and differentiation of HSCs. Second, vascular cells including endothelial cells and PVCs were found to express soluble factors that promote hematopoiesis and adhesion molecules such as E-selectin and VCAM1 that facilitate cell homing (21). Third, subpopulations of mesenchymal cells including CD146+ adventitial cells, CSCL12-abundant reticular cells, and adipocytes have been identified as $\mathrm{BM}$ niche components that regulate HSC function (22, 23). Among these cellular niches, PVCs have been consistently shown to function as a pivotal supporter in HSC maintenance (24, 25), illustrating the crosstalk of the PVCs and HSCs in the BM microenvironment. PVCs are one of the best contributors to maintaining HSCs in the BM, which can support LH-HSCs by binding with Notch ligand Jagged-1 $(26,27)$ suggesting close relationship with HSCs in reconstituting blood lineage cells. As shown in previous studies, hyperglycemia conditions resulted in the loss of PVCs in several tissues via accumulation of AGEs, high levels of VEGF, and ROS production $(28,29)$, suggesting relevance to vas- cular defects in diabetes. In our previous study, PVCs from the patients undergoing gestational diabetes showed significant defects in growth and regeneration potential (30). All these findings clearly indicate that hyperglycemic conditions influence the function and proliferation of HSCs by compromising the BM perivascular niche. Our results showed that the hyperglycemia-induced alteration in hematopoietic composition was partially recovered by the administration of PVC-CM. Thus, identification of specific regulatory soluble factors from PVCs is essential to apply disease-specific preconditioning treatments (31).

MDSCs originate from the myeloid lineage, but can be discriminated from other myeloid cell types by their immunosuppressive capacity. Their suppressive role is exerted through increased production of ARG1, NO and ROS in pathological conditions. We found that MDSC number and expression level of ARG1 and iNOS transcripts in the BM were significantly increased. These changes of MDSCs in the BM may play an important role in preventing or alleviating the progression of diabetic mellitus by suppressing $\mathrm{T}$ cell activity. Recent studies support the potential role of MDSCs in DM. For example, MDSCs are rapidly enriched in peripheral blood of both mice and human with T1DM $(32,33)$. Wang et al. (14) also showed that MDSCs prevent the development of T2DM by suppressing CD4+ $\mathrm{T}$ cell activity. These findings suggest that strategies to enhance MDSC suppressive function and number may be effective in preventing or alleviating DM.

Insulin signaling is a highly conserved metabolic process and diabetes accompanying with insulin resistance is caused by downregulation for the insulin receptor gene following persistent MAPK/ERK pathway inhibition (34). In addition, JNK and p38 are known as linked signal factors in diabetes $(35,36)$. Consistent with previous data, we also showed that phosphorylated ERK and p38 were activated in diabetic BM cells. However, and the levels of ERK and p38 were rapidly decreased by PVC-CM and DPI, implying that soluble factors from PVCs may be relevant to ERK and $\mathrm{p} 38$, but not JNK in restoring the microenvironment during diabetes. Further study is required to identify soluble factors from PVCs and define their roles in preventing or treating diabetic complications by reducing hematopoietic alterations in the BM.

\section{Acknowledgments}

This research was supported by grants from the Korea Health Technology R\&D Project through the Korea Health Industry Development Institute, funded by the Ministry of Health \& Welfare, Republic of Korea (Grant 
HI16C0047), the Ministry of Science, ICT and Future Planning (2015R1A4A1038666, 2016R1A2B4014890) and 2016 Research Grant from Kangwon National University (No.520160473).

\section{References}

1. Hossain P, Kawar B, El Nahas M. Obesity and diabetes in the developing world--a growing challenge. N Engl J Med 2007;356:213-215

2. Nagareddy PR, Murphy AJ, Stirzaker RA, Hu Y, Yu S, Miller RG, Ramkhelawon B, Distel E, Westerterp M, Huang LS, Schmidt AM, Orchard TJ, Fisher EA, Tall AR, Goldberg IJ. Hyperglycemia promotes myelopoiesis and impairs the resolution of atherosclerosis. Cell Metab 2013;17:695-708

3. Loomans CJ, de Koning EJ, Staal FJ, Rookmaaker MB, Verseyden C, de Boer HC, Verhaar MC, Braam B, Rabelink TJ, van Zonneveld AJ. Endothelial progenitor cell dysfunction: a novel concept in the pathogenesis of vascular complications of type 1 diabetes. Diabetes 2004;53:195-199

4. Fadini GP, Sartore S, Albiero M, Baesso I, Murphy E, Menegolo M, Grego F, Vigili de Kreutzenberg S, Tiengo A, Agostini C, Avogaro A. Number and function of endothelial progenitor cells as a marker of severity for diabetic vasculopathy. Arterioscler Thromb Vasc Biol 2006;26:21402146

5. Ferraro F, Lymperi S, Méndez-Ferrer S, Saez B, Spencer JA, Yeap BY, Masselli E, Graiani G, Prezioso L, Rizzini EL, Mangoni M, Rizzoli V, Sykes SM, Lin CP, Frenette PS, Quaini F, Scadden DT. Diabetes impairs hematopoietic stem cell mobilization by altering niche function. Sci Transl Med 2011;3:104ra101

6. Fadini GP, Fiala M, Cappellari R, Danna M, Park S, Poncina N, Menegazzo L, Albiero M, DiPersio J, StockerlGoldstein K, Avogaro A. Diabetes limits stem cell mobilization following G-CSF but not plerixafor. Diabetes 2015; 64:2969-2977

7. Han X, Deng Y, Yu J, Sun Y, Ren G, Cai J, Zhu J, Jiang G. Acarbose accelerates wound healing via Akt/eNOS signaling in $\mathrm{db} / \mathrm{db}$ mice. Oxid Med Cell Longev 2017;2017: 7809581

8. Kojima H, Kim J, Chan L. Emerging roles of hematopoietic cells in the pathobiology of diabetic complications. Trends Endocrinol Metab 2014;25:178-187

9. Kim H, Han JW, Lee JY, Choi YJ, Sohn YD, Song M, Yoon YS. Diabetic mesenchymal stem cells are ineffective for improving limb ischemia due to their impaired angiogenic capability. Cell Transplant 2015;24:1571-1584

10. Holmes D. Diabetes: SDF-1 dysregulation mediates diabetic stem cell mobilopathy. Nat Rev Endocrinol 2015;11: 318

11. Morohoshi M, Fujisawa K, Uchimura I, Numano F. Glucose-dependent interleukin 6 and tumor necrosis factor production by human peripheral blood monocytes in vitro.
Diabetes 1996;45:954-959

12. Guha M, Bai W, Nadler JL, Natarajan R. Molecular mechanisms of tumor necrosis factor alpha gene expression in monocytic cells via hyperglycemia-induced oxidant stressdependent and -independent pathways. J Biol Chem 2000; 275:17728-17739

13. Kim H, Cho HJ, Kim SW, Liu B, Choi YJ, Lee J, Sohn YD, Lee MY, Houge MA, Yoon YS. CD31+ cells represent highly angiogenic and vasculogenic cells in bone marrow: novel role of nonendothelial CD31+ cells in neovascularization and their therapeutic effects on ischemic vascular disease. Circ Res 2010;107:602-614

14. Wang T, Wen Y, Fan X. Myeloid-derived suppressor cells suppress CD4 $+\mathrm{T}$ cell activity and prevent the development of type 2 diabetes. Acta Biochim Biophys Sin (Shanghai) $2018 ; 50: 362-369$

15. Movahedi K, Guilliams M, Van den Bossche J, Van den Bergh R, Gysemans C, Beschin A, De Baetselier P, Van Ginderachter JA. Identification of discrete tumor-induced myeloid-derived suppressor cell subpopulations with distinct T cell-suppressive activity. Blood 2008;111:4233-4244

16. Zhou Z, French DL, Ma G, Eisenstein S, Chen Y, Divino CM, Keller G, Chen SH, Pan PY. Development and function of myeloid-derived suppressor cells generated from mouse embryonic and hematopoietic stem cells. Stem Cells 2010;28:620-632

17. Gabrilovich DI, Nagaraj S. Myeloid-derived suppressor cells as regulators of the immune system. Nat Rev Immunol 2009;9:162-174

18. Bronte V, Zanovello P. Regulation of immune responses by L-arginine metabolism. Nat Rev Immunol 2005;5:641-654

19. Schofield R. The relationship between the spleen colony-forming cell and the haemopoietic stem cell. Blood Cells 1978;4:7-25

20. Mercier FE, Ragu C, Scadden DT. The bone marrow at the crossroads of blood and immunity. Nat Rev Immunol 2011; 12:49-60

21. Rafii S, Mohle R, Shapiro F, Frey BM, Moore MA. Regulation of hematopoiesis by microvascular endothelium. Leuk Lymphoma 1997;27:375-386

22. Sacchetti B, Funari A, Michienzi S, Di Cesare S, Piersanti S, Saggio I, Tagliafico E, Ferrari S, Robey PG, Riminucci $M$, Bianco P. Self-renewing osteoprogenitors in bone marrow sinusoids can organize a hematopoietic microenvironment. Cell 2007;131:324-336

23. Sugiyama T, Kohara $H$, Noda $M$, Nagasawa T. Maintenance of the hematopoietic stem cell pool by CXCL12CXCR4 chemokine signaling in bone marrow stromal cell niches. Immunity 2006;25:977-988

24. Ding L, Saunders TL, Enikolopov G, Morrison SJ. Endothelial and perivascular cells maintain haematopoietic stem cells. Nature 2012;481:457-462

25. Kunisaki Y, Bruns I, Scheiermann C, Ahmed J, Pinho S, Zhang D, Mizoguchi T, Wei Q, Lucas D, Ito K, Mar JC, Bergman A, Frenette PS. Arteriolar niches maintain haematopoietic stem cell quiescence. Nature 2013;502:637-643 
26. Corselli M, Chin CJ, Parekh C, Sahaghian A, Wang W, Ge S, Evseenko D, Wang X, Montelatici E, Lazzari L, Crooks GM, Péault B. Perivascular support of human hematopoietic stem/progenitor cells. Blood 2013;121:2891-2901

27. Levesque JP. A niche in a dish: pericytes support HSC. Blood 2013;121:2816-2818

28. Hammes HP, Lin J, Renner O, Shani M, Lundqvist A, Betsholtz C, Brownlee M, Deutsch U. Pericytes and the pathogenesis of diabetic retinopathy. Diabetes 2002;51: 3107-3112

29. Warmke N, Griffin KJ, Cubbon RM. Pericytes in diabetesassociated vascular disease. J Diabetes Complications 2016; 30:1643-1650

30. An B, Kim E, Song H, Ha KS, Han ET, Park WS, Ahn TG, Yang SR, Na S, Hong SH. Gestational diabetes affects the growth and functions of perivascular stem cells. Mol Cells 2017;40:434-439

31. Ranganath SH, Levy O, Inamdar MS, Karp JM. Harnessing the mesenchymal stem cell secretome for the treatment of cardiovascular disease. Cell Stem Cell 2012;10:244-258
32. Whitfield-Larry F, Felton J, Buse J, Su MA. Myeloid-derived suppressor cells are increased in frequency but not maximally suppressive in peripheral blood of Type 1 Diabetes Mellitus patients. Clin Immunol 2014;153:156-164

33. Hassan M, Raslan HM, Eldin HG, Mahmoud E, Elwajed HAA. CD33 + HLA-DR- myeloid-derived suppressor cells are increased in frequency in the peripheral blood of Typel diabetes patients with predominance of CD14+ subset. Open Access Maced J Med Sci 2018;6:303-309

34. Zhang W, Thompson BJ, Hietakangas V, Cohen SM. MAPK/ERK signaling regulates insulin sensitivity to control glucose metabolism in Drosophila. PLoS Genet 2011; 7:e1002429

35. Igarashi $\mathrm{M}$, Wakasaki $\mathrm{H}$, Takahara N, Ishii H, Jiang ZY, Yamauchi T, Kuboki K, Meier M, Rhodes CJ, King GL. Glucose or diabetes activates p38 mitogen-activated protein kinase via different pathways. J Clin Invest 1999;103:185195

36. Bennett BL, Satoh Y, Lewis AJ. JNK: a new therapeutic target for diabetes. Curr Opin Pharmacol 2003;3:420-425 